\title{
COMPUTED TOMOGRAPHY OF RENAL ONCOCYTOMAS
}

\author{
Robert W. Jasinski, ${ }^{1}$ Marco A. Amendola, ${ }^{1 *}$ Gary M. Glazer, ${ }^{1}$ \\ Robert L. BreE ${ }^{2}$ and PaUl W. Gikas ${ }^{3}$ \\ ${ }^{1}$ Department of Radiology and ${ }^{3}$ Department of Pathology, University of Michigan Hospital, Ann Arbor, \\ MI 48109, U.S.A. and ' 2 epartment of Radiology, Beaumont Hospital, Royal Oak, MI 48072, U.S.A
}

(Received 3 January 1985; in revised form 2 April 1985; received for publication 22 April 1985)

\begin{abstract}
A retrospective analysis of 5 renal oncocytomas studied with $\mathrm{CT}$ was performed. Oncocytomas appeared as rounded hypodense solid masses with sharp margination from the normal renal parenchyma. They showed uniform homogeneous enhancement on CT scans performed after drip infusion of urographic contrast. One oncocytoma studied with dynamic CT scanning demonstrated a stellate (spoke-wheel) pattern of enhancement. No evidence of involvement of adjacent renal parenchyma, perinephric fat, renal veins or regional lymph nodes was present at $\mathrm{CT}$ and the findings were confirmed at surgery. Although the differential diagnosis between renal oncocytoma and hypernephroma may not be possible, preoperative awareness of a potentially benign lesion may guide the surgeon to attempt renal preserving surgery instead of the customary nephrectomy.
\end{abstract}

Kidney, neoplasms Oncocytoma Hypernephroma, computed tomography Contrast enhancement

\section{INTRODUCTION}

Renal oncocytoma is a benign neoplasm which ideally should be preoperatively distinguished from renal cell carcinoma in an effort to conserve renal tissue at surgery [1]. Arteriography has been suggested as capable of diagnosing oncocytoma, however, arteriographically, renal cell carcinoma and oncocytoma can be very similar [2-4]. Excretory urography, radionuclide scanning, and ultrasound are nonspecific [2-6]. Furthermore, needle biopsy is not reliable because the variegated histologic character of renal cell carcinoma may mimic oncocytoma on the basis of a limited sample [7]. In this report we present the CT findings in five renal oncocytomas.

\section{CASE REPORTS}

\section{Case I}

A 63-year-old man was admitted to an outside hospital with the chief complaint of nocturia. Excretory urography revealed bilateral renal masses which were solid on subsequent ultrasound examination. Arteriography confirmed bilateral renal masses with vascular encasement in the right one suggesting renal cell carcinoma. Technetium ${ }^{99 m}$ glucoheptonate scan showed a cold area in each kidney corresponding to the known renal masses. The patient was referred to the University of Michigan Hospital where body CT was performed. Precontrast CT showed a mass in each kidney, each mass slightly less dense than the normal renal parenchyma. Postcontrast infusion views revealed homogeneous well circumscribed masses in each [Fig. 1(A)]. Dynamic scanning with bolus injection revealed a normal left renal vein and a "spoke-wheel" appearance in the left renal oncocytoma [Fig. 1(B)]. The $3 \mathrm{~cm}$ right sided lesion showed homogenous enhancement at dynamic CT. The remainder of the CT exam showed no evidence of extrarenal extension, liver metastases or lymph node involvement. The patient was taken to surgery where frozen section of the left sided mass showed oncocytoma and partial left nephrectomy was performed. On the right a radical nephrectomy was performed without prior biopsy. Final histology indicated that both tumors were oncocytomas. No evidence of renal vein or nodal involvement was present at operation. Macroscopic exam of the left sided mass showed a well circumscribed brown tumor with a $1 \mathrm{~mm}$ fibrous pseudocapsule. Gross exam of the right sided mass showed an ovoid well circumscribed nodule bulging above the capsular surface,

${ }^{*}$ Address correspondence and reprint requests to: Marco A. Amendola. M.D., Department of Radiology, Box 13, University Hospital, Ann Arbor, MI 48109, U.S.A. 


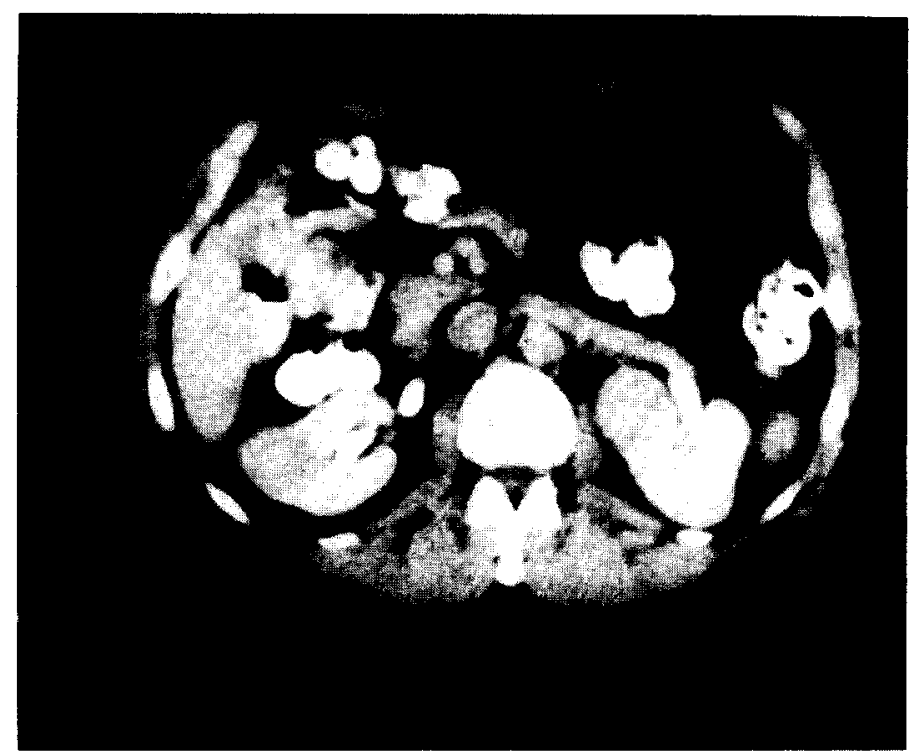

(A)

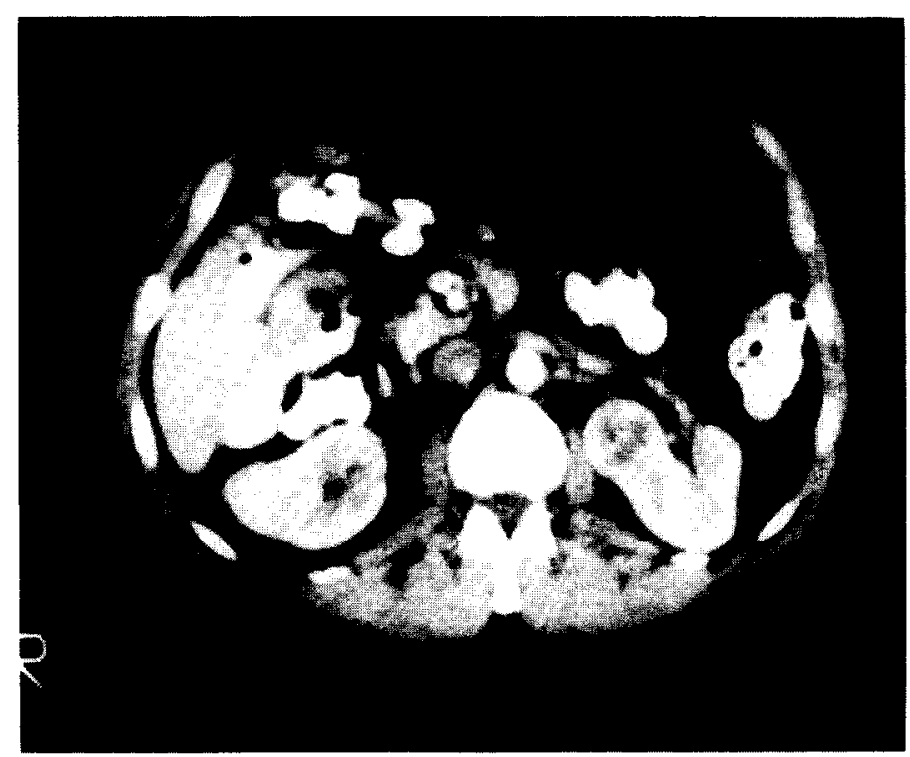

(B)

Fig. 1.(A) Case 1. CT scans in a 63-year-old man with bilateral oncocytomas: Postcontrast CT showing uniformly hypodense masses in each kidney. Both are well circumscribed and sharply marginated. (B) Scan through both oncocytomas during a bolus injection of contrast demonstrates a "spoke-wheel" stellate appearance in the left sided tumor.

appearing to penetrate the renal capsule, but without extension to the adipose tissue. The cut surface of the tumor had a light brown color. Electron microscopy revealed the ultrastructural features of an oncocytoma. On three year clinical and radiographic follow-up, there is no evidence of recurrence or metastatic disease.

\section{Case 2}

A 34-year-old female was evaluated for back pain by CT which demonstrated an incidental left upper pole renal mass measuring $4 \mathrm{~cm}$ in diameter. The mass was slightly hypodense compared to the normal kidney on precontrast scan [Fig. 2(A)]. Dynamic scanning showed a well circumscribed lesion without evidence for perinephric or extrarenal extension [Fig. 2(B)]. The mass enhanced homogeneously, but to a lesser degree than the adjacent renal parenchyma from which it was separated by a fine rim of enhancement. No abdominal metastases or renal vein involvement were 


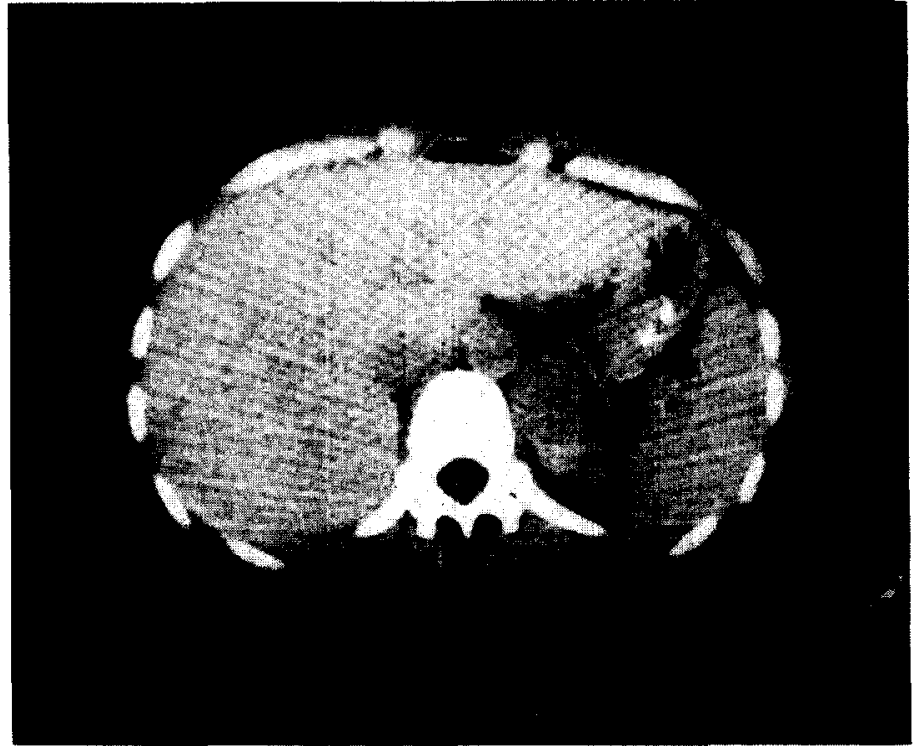

(A)

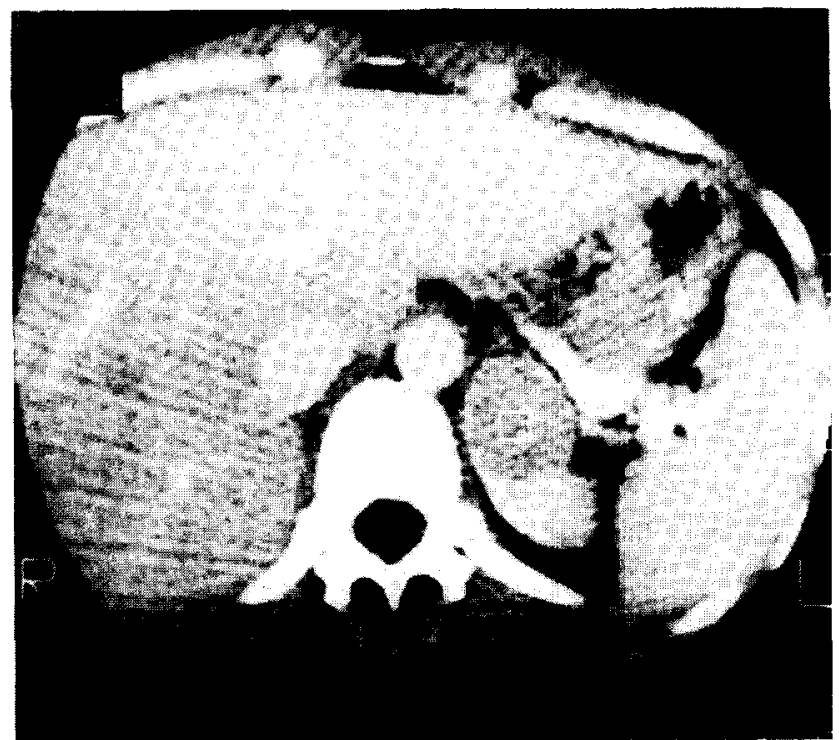

(B)

Fig. 2.(A) Casc 2. Prccontrast CT in a 34-year-old female: hypodense mass in the upper pole of the left kidney. (B) Dynamic CT scanning during bolus injection of contrast. Homogeneously enhancing left renal oncocytoma (cursor: 95 H.U.), hypodense with respect to normal renal parenchyma, from which it is separated by a fine rim of enhancement.

identified. Intraoperatively the surgeon could not determine on gross inspection whether this was a benign or malignant lesion, however, no extrarenal disease was identified. A left nephrectomy revealed a renal oncocytoma on permanent section.

\section{Case 3}

A 64-year-old female with a history of recurrent pancreatitis was admitted for abdominal pain. A noncontrast CT incidentally demonstrated a $5 \mathrm{~cm}$ hypodense well demarcated left renal mass with density of 23 H.U. not consistent with a simple cyst (Fig. 3). No intravenous contrast was given because of history of severe reaction to urographic contrast in the past. Sonography confirmed the solid nature of the mass. At operation, a brown neoplasm was identified in the left kidney. Gross pathology demonstrated a well circumscribed tumor with a central white stellate scar and focal capsular hemorrhage, but without necrosis or hemorrhage within the mass. No invasion of the renal pelvis or renal vein was present. Histologic diagnosis was renal oncocytoma. Review of microscopy 


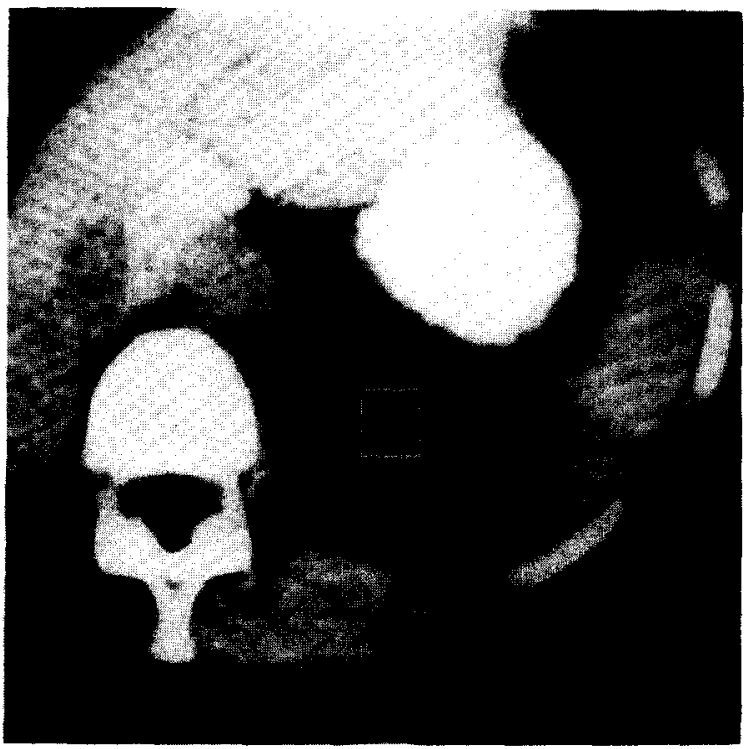

Fig. 3. Case 3. Renal oncocytoma incidentally discovered in a 64-year-old female: precontrast CT scan shows well demarcated, homogeneously hypodense left upper pole renal mass (cursor: 23 H.U.).

slides demonstrated alternating areas of oncocytes in tubular arrangement and relatively avascular regions of fibrous tissue.

\section{Case 4}

During work-up of chronic right upper quadrant pain in a 50-year-old female, ultrasound showed a right renal mass which was hyperechoic suggesting the possibility of angiomyolipoma. At CT there was a $4 \mathrm{~cm}$ solid mass without evidence for fat (Fig. 4). No extrarenal disease was identified at CT. Angiography demonstrated a vascular mass with intense mottled stain on the nephrographic phase. A right nephrectomy was performed. No evidence for metastatic disease was identified. Cut section revealed a yellow-tan tumor which was well circumscribed, but not encapsulated. Histology indicated that the tumor was an oncocytoma.

\section{DISCUSSION}

Renal oncocytoma is a neoplasm whose character is a matter of controversy, although most authorities favor a benign lesion [8-14]. The presence of oncocytes in normal organs, as a sometimes

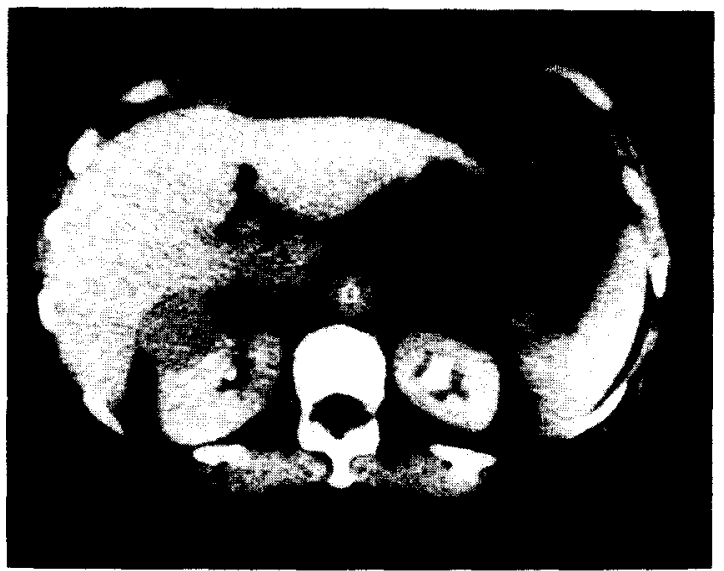

Fig. 4. Case 4. Right renal oncocyloma in a 50-year-old female: Postcontrast infusion CT scan demonstrating a mildly enhanced mass with sharp margination and of homogeneous appearance less dense than the surrounding normal kidney. 
substantial portion of well documented hypernephromas, and in tumors in other portions of the body such as salivary glands, thyroid, parathyroid, and adrenal gland where their behavior at times is clearly malignant [8], contributes to the controversy. Even those authors who regard oncocytoma as a distinct entity, acknowledge the potential for confusion with renal carcinoma on histologic grounds [15]. For a tumor to be called an oncocytoma, it must be entirely composed of oncocytes to avoid confusion with renal carcinoma which may contain some areas with oncocytic features [8-12]. Although the preoperative diagnosis of renal oncocytoma has been reported using aspiration biopsy techniques [16], the requirement that all portions of the neoplasm be composed of oncocytes limits the accuracy of percutaneous biopsy diagnosis $[1,6-7]$.

The size range of the oncocytoma is from 1 to $13 \mathrm{~cm} \mathrm{[10]} \mathrm{with} \mathrm{average} \mathrm{sizes} \mathrm{being} 5-7 \mathrm{~cm}$ in greatest diameter $[3,17]$. It has an approximate 2 to 1 male to female predilection [10]. The age range includes the 4 th to 10 th decades with a peak at the 5th-8th decades $[5,11]$. This unfortunately parallels the age and sex predilection of renal cell carcinoma [18]. Multiple and bilateral oncocytomas have been described previously and the lesion is equally distributed between both kidneys $[3,5]$. As in our patients, the neoplasm is often merely an incidental finding on examinations performed for other indications [6, 13]. Unlike renal carcinoma, oncocytoma has an excellent prognosis [2, 19-21].

Oncocytoma is of importance to the radiologist because it mimics renal cell carcinoma [13]. On ultrasound, oncocytoma cannot be distinguished from hypernephroma since it appears as a solid mass with echogenicity similar to renal cortex. Radionuclide scanning while initially thought to be potentially useful [2], has been shown to be of no help in distinguishing renal carcinoma from oncocytoma [3,5]. Arteriography has been the only imaging technique of some value in recognizing the oncocytoma preoperatively $[2,17,19]$. The most characteristic findings are a spoke/wheel configuration, a homogeneous nephrogram similar to the normal nephrogram, a sharp well marginated lesion and absence of irregular tumor vessels, or arterio-venous shunting. While these findings are most suggestive of oncocytoma, many oncocytomas fail to have all of them and some of these angiographic characteristics are shared by approx $15 \%$ of renal cell carcinomas $[2,4]$.

The gross appearance of oncocytoma is distinct from that of renal carcinoma. Characteristic macroscopic findings in oncocytoma include a central fibrous scar with fibrous septae in the tumor producing a stellate appearance $[1,6,7]$, a homogeneous cut surface of tan brown color without evidence of hemorrhage or necrosis $[1,6,20]$, sharp demarcation with respect to adjacent structures, lack of perinephric involvement, lack of metastases and absence of venous invasion [1-3, 6, 7, 21, 22]. The connective tissue capsule which separates the tumor from normal renal parenchyma has been described as vascular rather than avascular [11]. This may explain the rim enhancement seen in our case II and also noted in a previous case studied by CT [23]. All reported cases of renal oncocytoma do not match perfectly with the above described gross appearance $[5,11,15-17,20]$, but discrepancy is present in only a minority of cases.

On contrast enhanced CT, oncocytoma has been reported to appear as a homogeneous well demarcated solid renal mass slightly less dense than the renal cortex [3, 5, 24]. This has also been our experience. However in one of our cases (Fig. 1) dynamic CT revealed a pattern suggestive of a "spoke wheel". This has not been previously described to our knowledge. The spoke wheel seen in this case at CT may represent the stellate appearance of the fibrous scar classically described in the gross specimen. Although in this particular case of a $3 \mathrm{~cm}$ oncocytoma (Fig. 1), a stellate scar was not seen at gross inspection, on microscopy, areas of fibrous stranding were clearly evident within the tumor. Our largest oncocytoma $(5 \mathrm{~cm})$ had a stellate scar on gross exam. Unfortunately, enhanced CT scans were not obtained due to history of contrast reaction. We and others [24] have observed that in small oncocytomas in which the stellate scar is not identifiable at gross pathology, the tumors are homogeneous but less dense than normal kidney tissue after intravenous infusion. In 2 previously reported cases, a central low density area has been demonstrated on enhanced $\mathrm{CT}$, corresponding to a stellate scar on gross exam. Both oncocytomas were rather large, one measuring $5 \mathrm{~cm}$ and the other $6 \mathrm{~cm}$ in size $[25,26]$. It is likely that large size oncocytomas with a central fibrous stellate scar will have a central lucent area on CT exam corresponding to this avascular region unlike small oncocytomas which will appear homogeneous.

At variance with previous reports [24], in our oncocytomas a homogeneous hypodense appearance was consistently present on precontrast CT. However, attenuation values greater than $20 \mathrm{H} . \mathrm{U}$. allowed differentiation from a simple renal cyst in all cases. There has been only a single reported 


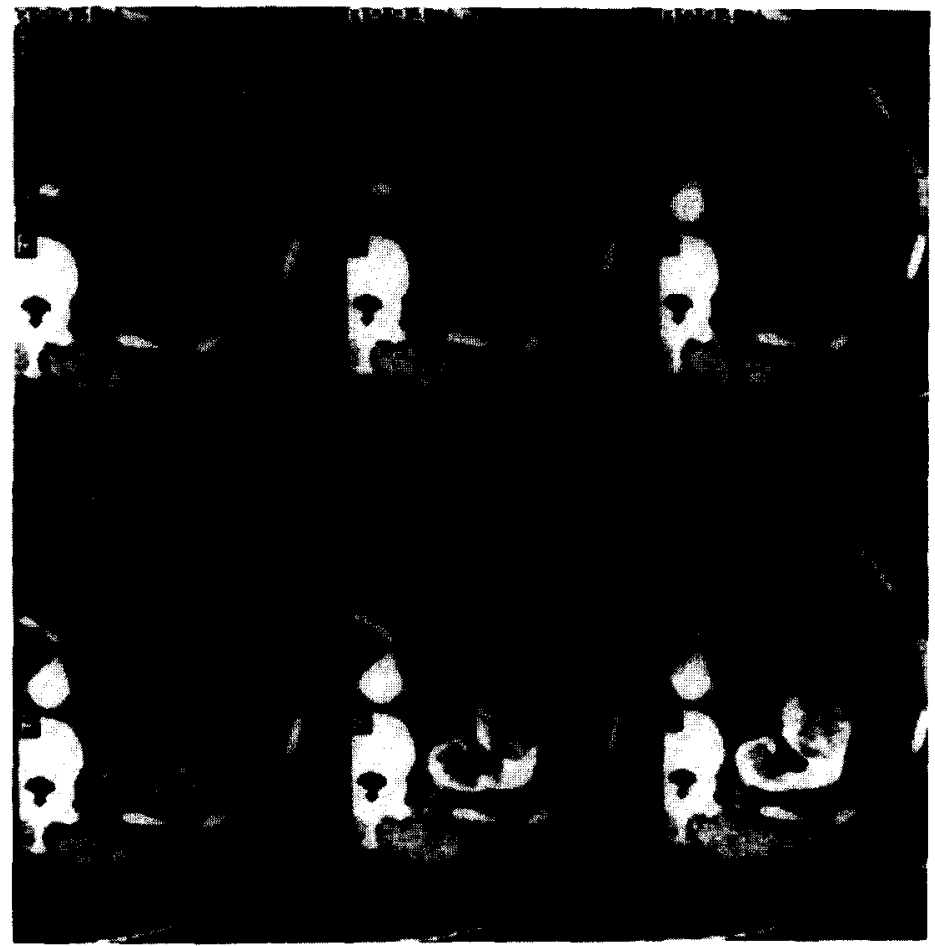

Fig. 5. Dynamic CT of a $3 \mathrm{~cm}$ hypernephroma with bolus injection demonstrating precontrast inhomogeneity, followed by irregular enhancement and a thick margin, quite unlike renal oncocytoma.

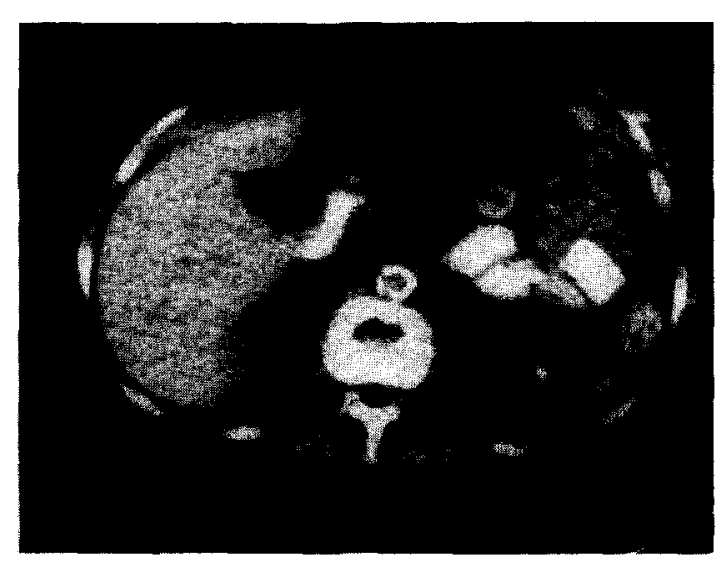

(A)

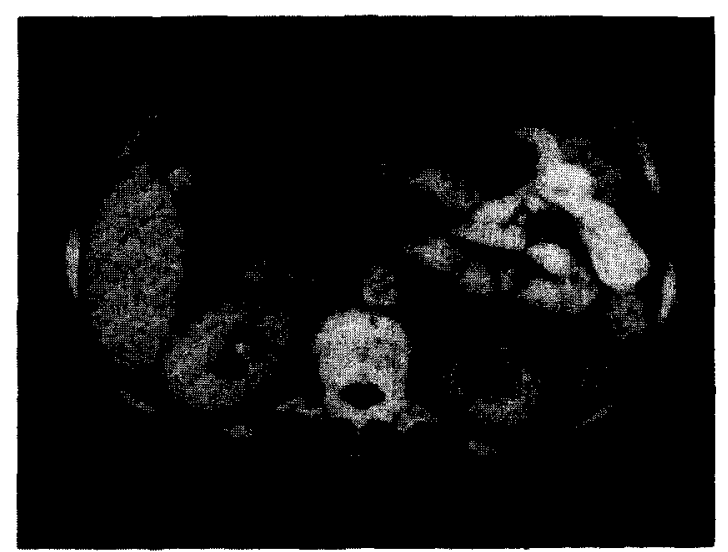

(B)

Fig. 6.(A) Small hypernephroma slightly less dense than remainder of the left kidney on precontrast CT. Note a small calcification in its margin. (B) Mass is hypodense and fairly well circumscribed in post contrast views closely simulating an oncocytoma. 
instance of an oncocytoma denser than normal kidney in precontrast CT [27]. In our series the oncocytomas were homogeneously hypodense post contrast unlike most hypernephromas (Fig. 5). No oncocytoma showed calcification in our scries. Although calcification present at CT is not rare in hypernephromas [Fig. 6(A)], only once has calcification been reported in oncocytoma [28]. The presence of sharp margination does not absolutely exclude hypernephroma, although only a minority of hypernephromas show sharp margination [Fig. 6(B)].

Our data suggest that it may be possible to suggest the diagnosis of oncocytoma using CT . Preoperative awareness of a potentially benign lesion may guide the surgeon to careful evaluation of the gross and microscopic features of the tumor hopefully resulting in renal preserving surgery instead of the customary nephrectomy.

\section{SUMMARY}

Our series of five surgically proven renal oncocytomas and a review of the literature suggest that some features of a solid renal mass studied by CT should alert the radiologist to the possibility of dealing with a renal oncocytoma. These include a well marginated mass, absence of calcification and homogeneous, hypodense appearance on precontrast CT. Following contrast infusion if the mass is less than $5 \mathrm{~cm}$ in size it is likely to enhance homogeneously (but to a lesser degree than normal cortex) and if larger, may present a central lower attenuation area corresponding to the scar found on gross pathologic exam. In one our oncocytomas studied with dynamic CT during a bolus injection of contrast, an intriguing "spoke-wheel" pattern was seen. The significance and specificity of this observation is not clear at this time but greater use of dynamic CT scanning in the evaluation of renal masses is likely to clarify this issue in the near future.

\section{REFERENCES}

1. M. J. Klein and Q. J. Valensi, Proximal tubular adenoma of kidney with so-called oncocytic features, Cancer 38, 906 (1976)

2. M. A. Ambos, M. A. Bosniak, Q. J. Valensi, M. A. Madayag and R. S. Lefleur, Angiographic patterns in renal oncocytomas, Radiology 129, 615 (1978).

3. J. A. Bonavita, H. M. Pollack and M. P. Banner, Renal oncocytoma: Further observations and literature review, Urol. Radiol. 2, 229 (1981).

4. R. A. Older, D. M. Cleeves, B. F. Fetter and D. A. Jackson, "Spoke-wheel" angiographic pattern in renal masses: nonspecificity (letter to the editor), Radiology 128, 836 (1978).

5. E. M. Lautin, A. C. Friedman, P. M. Gordon, J. F. McCormick, F. B. Fromowitz and M. S. Goldman, Radionuclide imaging and computed tomography in renal oncocytoma, Radiology 138, 185 (1981)

6. A. Morales, S. Wasan and S. Bryniak, Renal oncocytomas: clinical, radiological, and histological features, J. Urol. 123, $261(1980)$.

7. G. S. M. Yu, S. Rendler, A. Herkowitz and J. J. Molnar, Renal oncocytoma, Cancer 45, 1010 (1980).

8. H. Hamperl, Benign and malignant oncocytoma, Cancer 15, 1019 (1962).

9. H. P. Jander, Renal oncocytoma: a nonentity (letter to the editor), Radiology 130, 815 (1979).

10. A. M. Tessler, S. Kurusu, M. J. Klein and Q. J. Valensi, Proximal tubular adenoma of kidney, Urology 10, 203 (1977).

11. A. P. Chaudhry, S. K. Satchidanand, J. F. Gaeta, E. Slotkin, S. Shenoy and P. A.Nickerson, Light and ultrastructural studies of renal oncocytic adenoma, Urology 14, 392 (1979).

12. M. M. Lieber, K. M. Tomera and G. M. Farrow, Renal oncocytoma, J. urol. 125, 481 (1981).

13. H. Choi, U. A. Almagro, J. T. McManus, D. H. Norback and S. C. Jacobs, Renal oncocytoma, a clinicopathologic study, Cancer 51, 1887 (1983).

14. J. D. Van DerWalt, H. A. S. Reid, A. R. Risdon and J. H. F. Shaw, Renal oncocytoma. A review of the literature and report of an unusual multicentric case, Virchows Arch. 398, 291 (1983).

15. M. Akhtar and E. Kott, Oncocytoma of kidney, Urology 14, 397 (1979).

16. C. A. Rodriguez, A. Buskop, J. Johnson, F. Fromowitz and L. G. Koss, Renal oncocytoma: preoperative diagnosis by aspiration biopsy, Acta cytol. 24, 355 (1980).

17. S. N. Weiner and R. G. Bernstein, Renal oncocytoma: Angiographic features of 2 cases, Radiology 125, 633 (1977).

18. A. R. Kendall, Incidentally found renal mass, J. Urol. 124, 269 (1980).

19. T. A. Sos, G. F. Gray and H. A. Baltaxe, The angiographic appearance of benign renal oxyphilic adenoma, Am. J. Roentg. 127,717 (1976).

20. J. R. Johnson, Oncocytoma of kidney, Urology 14, 181 (1979).

21. H. D. Pearse and D. C. Houghton, Renal oncocytoma, Urology 13, 74 (1979).

22. G. Pillari, W. J. Lee, S. Kumari et al., CT and angiographic correlates: surgical image of renal mass lesions, Urology 17, $296(1981)$.

23. K. H. Barth and M. Menon, Renal oncocytoma. Further diagnostic observations, Diagn. Imaging 49, 259 (1980).

24. E. Levine and M. Huntrakoon, Computed tomography of renal oncocytoma, Am. J. Roentg. 141, 741 (1983).

25. R. H. Cohan, N. R. Dunnick, G. E. Degesys and M. E. Korobkin, Computed tomography of renal oncocytoma, J. Comput. assist. Tomogr. 8, 284 (1984).

26. J. Fraga, Y. Mairy, C. Fievez, Marbaixe and P. Hennebert, L'oncocytome renal, Acta urol. belg. 50, 301 (1982). 
27. J. Wojtowicz, А. Karkowski, J. Konkiewicz and B. Lukaszewski, Renal oncocytoma, J. Comput. assist. Tomogr. 3, 124 (1979)

28. N. F. Wasserman and S. L. Ewing, Calcified renal oncocytoma, Am. J. Roentg. 141, 747 (1983).

About the Author-RoberT W. JASINSKI received his M.D. from the University of Chicago, Pritzker School of Medicine. Following radiology residency in the University of Chicago he was a fellow in body computed tomography and ultrasonography at the University of Michigan, Ann Arbor. He is currently a fellow in Angiography and Interventional Radiology at Loma Linda University, California.

About the Author-Marco A. AmEndola received his M.D. from the University of Republic of Uruguay, Montevideo. He completed his radiology residency and fellowship in Computed Tomography at Albert Einstein Medical Center, Philadelphia. He is currently Associate Professor of Radiology, and Co-Director Division of Abdominal Radiology at the University of Michigan.

About the Author--GaRY M. GLAZER received his M.D. from Case Western Reserve University. He completed radiology residency and fellowship training (body computed tomography and ultrasonography) at the University of California, San Francisco. He is currently Associatc Professor and Director, Division of Body Computed Tomography at the University of Michigan.

About the Author-Robert L. BreE received an M.D. degree from the University of Michigan, Ann Arbor. He was trained in radiology at St. Joseph's Hospital and Medical Center, Phoenix, Arizona, and at the University of Michigan Medical Center. He is Director of the Division of Ultrasound at William Beaumont Hospital, Royal Oak, Michigan, Department of Radiology, and Clinical Associate Professor of Radiology at the University of Michigan.

About the Author-Paul W. GiKas received his M.D. from the University of Michigan. He is currently Professor of Pathology at the University of Michigan. 